\title{
ATLAS Jet Reconstruction, Calibration, and Tagging of Lorentz- boosted Objects
}

\author{
Steven Schramm ${ }^{1}$, on behalf of the ATLAS Collaboration \\ ${ }^{1}$ Université de Genève $(\mathrm{CH})$
}

\begin{abstract}
Jet reconstruction in the ATLAS detector takes multiple forms, as motivated by the intended usage of the jet. Different jet definitions are used in particular for the study of QCD jets and jets containing the hadronic decay of boosted massive particles. These different types of jets are calibrated through a series of mostly sequential steps, providing excellent uncertainties, including a first in situ calibration of the jet mass scale.

Jet tagging is investigated, including both not-top-quark vs gluon discrimination as well as $W / Z$ boson, $H \rightarrow b b$, and top-quark identification. This includes a first look at the use of Boosted Decision Trees and Deep Neural Networks built from jet substructure variables, as well as Convolutional Neural Networks built from jet images. In all cases, these advanced techniques are seen to provide gains over the standard approaches, with the magnitude of the gain depending on the use case. Future methods for improving jet tagging are briefly discussed, including jet substructure-oriented particle flow primarily for $W / Z$ tagging and new subjet reconstruction strategies for $H \rightarrow b b$ tagging.
\end{abstract}

\section{Introduction}

During the 2015 to 2017 data-taking period, the Large Hadron Collider (LHC) [1] has collided protons at a center of mass energy of $\sqrt{s}=13 \mathrm{TeV}$ and at an instantaneous luminosity drastically surpassing the design value of $1 \times 10^{34} \mathrm{~cm}^{-2} \mathrm{~s}^{-1}$. The vast majority of the inelastic proton-proton collisions which occur at the LHC ultimately result in the production of quarks and gluons, which cannot exist in isolation due to colour confinement. As a result, the quarks and gluons undergo hadronization, and are observed in the ATLAS [2] detector as collimated streams of particles depositing energy in the calorimeters. These collections of calorimeter energy deposits are reconstructed as jets, which are a useful way to represent the hadronic energy present in a given proton-proton collision.

While jets are a useful tool, they are only a representation of the underlying physical process, and different use cases benefit from the use of different jet definitions. ATLAS primarily makes use of small- $R$ jets for physics results with quarks and gluons, while large- $R$ jets provide an alternative interpretation of the same event which is beneficial for studies of hadronically decaying massive particles. In both cases, the resulting jets must be calibrated in order to provide an accurate representation of the event, and then the jets can be tagged to understand their likely originating particle.

The ATLAS detector will be briefly discussed in Section 2, followed by the details of jet reconstruction in Section 3. Section 4 describes the various stages of the ATLAS jet calibration procedure, while Section 5 demonstrates the use of jet tagging techniques, especially as applied to Lorentzboosted objects. A brief conclusion is provided in Section 6. 


\section{Detector}

The ATLAS detector is nearly hermetic and provides full $4 \pi$ angular coverage ${ }^{1}$ for particles produced in LHC collisions. The detector consists of three concentric sensitive volumes, the innermost of which is the Inner Detector (ID). The ID is immersed in a $2 \mathrm{~T}$ axial magnetic field, provided by a solenoid, and enables precision track reconstruction for $|\eta|<2.5$. The ID is enclosed by layers of electromagnetic and hadronic calorimeters, covering the full range of $|\eta|<4$.9. The electromagnetic calorimeters all use Liquid Argon (LAr) as the sensitive material, while lead is used as the absorber in the central $(|\eta|<1.475)$ and endcap $(1.375<|\eta|<3.2)$ regions. The hadronic calorimeters are divided into the barrel $(|\eta|<0.9)$, extended barrel $(0.8<|\eta|<1.7)$, and endcap $(1.5<|\eta|<3.2)$ regions. The barrel and extended barrel make use of scintillating tiles as the active medium and steel for the absorber, while the endcap uses a LAr sensitive material with copper absorbers. Electromagnetic and hadronic energy measurements are provided in the forward region $(3.1<|\eta|<4.9)$ using a LAr sensitive volume and copper or tungsten absorbers respectively. The calorimeter itself is enclosed by the Muon Spectrometer (MS), providing precision muon track measurements for $|\eta|<2.7$, within a magnetic field provided by three large air-core toroid magnets.

Typical LHC collisions consist of many proton-proton interactions within a single bunch crossing, averaging roughly 30 simultaneous interactions on top of the hard-scatter collision of interest during the 2015 to 2017 data taking period. These additional interactions are referred to as in-time pile-up, and generally manifest as low-energy diffractive events, depositing only a small amount of energy in the ATLAS calorimeters. The calorimeters using LAr as the sensitive volume are also particularly sensitive to out-of-time pile-up, which is the result of signals from previous or subsequent bunch crossings appearing in the readout of the crossing of interest. This is possible as the space between subsequent bunch crossings is much smaller than the amount of time it takes to read out the calorimeter, thus residual signals from adjacent crossings pollute the signal of interest. The LAr calorimeters use pulse shapes that negate this effect on average, but residual local fluctuations remain significant, especially as the average number of collisions per bunch crossing increases.

Both of these two sources of additional energy in the calorimeter must be addressed, particularly as the average number of collisions per bunch crossing continues to increase. These pile-up contributions serve to add a layer of noise to the detector, which must be removed to study the behaviour of the underlying event of interest.

\section{Jet reconstruction}

Jet reconstruction can be divided into three primary stages: first the input four-vectors are defined, then the jet algorithm and parameters are defined, and finally the jet is groomed (for large- $R$ jets).

\subsection{Jet inputs}

ATLAS primarily uses topo-clusters as the inputs to jet reconstruction, where topo-clusters are topologically-grouped noise-suppressed clusters of calorimeter cells. Topo-clusters are formed from seed cell(s) with more than $4 \sigma$ of energy, where $\sigma$ is the average amount of noise expected in the cell in question, defined as the sum of the expected electronic and pile-up noise [3]. In current data-taking conditions, the pile-up noise dominates over the electronic noise. All cells adjacent to the seed cell(s)

\footnotetext{
${ }^{1}$ ATLAS uses a right-handed coordinate system with its origin at the nominal interaction point (IP) in the centre of the detector and the $z$-axis along the beam pipe. The $x$-axis points from the IP to the centre of the LHC ring, and the $y$-axis points upward. Cylindrical coordinates $(r, \phi)$ are used in the transverse plane, $\phi$ being the azimuthal angle around the $z$-axis. The pseudorapidity is defined in terms of the polar angle $\theta$ as $\eta=-\ln \tan (\theta / 2)$.
} 
in three dimensions are then grouped together, so long as they have at least $2 \sigma$ of energy, and this process repeats until there are no such adjacent cells. The process concludes by adding all calorimeter cells adjacent to the topo-cluster, irrespective of their energy.

Topo-clusters can be calibrated at either the raw (electromagnetic, EM) scale, where the energy of an isolated topo-cluster is the sum of its constituent cell energies, or at the local cell weighting (LCW) scale. The LCW scale accounts for the difference between electromagnetic and hadronic interactions in the ATLAS calorimeters, thereby correcting the average topo-cluster to the hadronic energy scale.

Due to the way in which topo-clusters are formed, they are naturally pile-up suppressed. However, this form of pile-up suppression is designed for the average topo-cluster in average expected datataking conditions. By taking advantage of event-by-event measurements of the pile-up levels, as well as local observables, it is possible to further reduce the pile-up dependence of topo-clusters. Several different techniques have been studied, and have been shown to improve the low- $p_{\mathrm{T}}$ jet resolution, where pile-up noise significantly impacts the jet energy resolution. The gain is seen to be small in current pile-up conditions, but to grow to up to a $30 \%$ reduction for jets with $p_{\mathrm{T}} \approx 30 \mathrm{GeV}$ once the average number of collisions per bunch crossing reaches 200 [4].

Despite these potential longer-term benefits, typical jets in ATLAS make use of standard topoclusters, using EM topo-clusters for small- $R$ jets and LCW topo-clusters for large- $R$ jets. Unless otherwise mentioned, this includes all of the following results in this document.

\subsection{Jet algorithms}

ATLAS typically uses the anti- $k_{t}$ [5] algorithm for jet reconstruction as provided by the FastJet [6] software library. Two different distance parameters $R$ are typically used, corresponding to different intended uses. Jets representing quarks and gluons are typically called small- $R$ jets, and are reconstructed with $R=0.4$. On the other hand, jets representing hadronically decaying massive particles are typically called large- $R$ jets, and are reconstructed with $R=1.0$.

In the latter case, the larger radius is useful to capture all of the decay products within a single jet, as the angular separation $\Delta R$ between the constituents of a massive particle $\chi$ undergoing a two-body decay follow $\Delta R \gtrsim 2 m^{\chi} / p_{\mathrm{T}}^{\chi}$, as seen in Figure 1. For multi-stage decays, such as $t \rightarrow b W \rightarrow b q q$, the decay products are less collimated, further necessitating the use of large distance parameters.

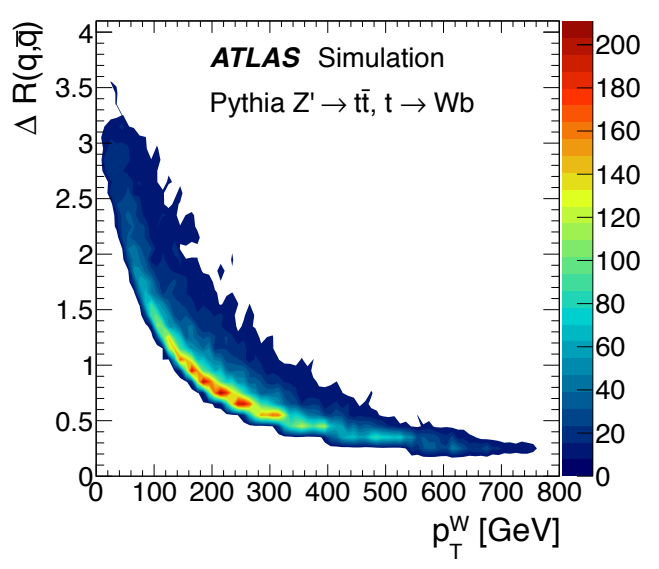

Figure 1. The separation $\Delta R$ between the pair of quarks from a hadronically decaying $W$ boson, demonstrating the two-body decay behaviour of $\Delta R \gtrsim 2 m^{\chi} / p_{\mathrm{T}}^{\chi}[7]$. 


\subsection{Jet grooming}

Using large- $R$ jets is necessary to fully contain the hadronic massive particle decays, but it comes with a substantially increased sensitivity to pile-up effects due to the larger fraction of the calorimeter enclosed within the jet volume. Additionally, while pile-up may be low energy and thus not change the total jet kinematics by a large amount, it is randomly distributed, and can thus obscure the angular structure within the jet that is the key to identifying massive particle decays.

To get around these limitations, large- $R$ jets are typically groomed, where grooming is a class of algorithms that take a jet and throw away constituents following a defined strategy, rebuilding the final jet from the remaining constituents. ATLAS typically uses the trimming procedure [8] with parameters of $f_{\text {cut }}=5 \%$ and $R_{\text {sub }}=0.2$. Trimming a jet consists of taking the original anti- $k_{t}$ jet, reclustering the constituents in it using the $k_{t}$ algorithm [9] with a distance parameter of $R_{\mathrm{sub}}$, and throwing away any constituents in a given $k_{t}$ sub-jet if the sub-jet $p_{\mathrm{T}}$ is less than $f_{\text {cut }}$ of the large- $R$ jet $p_{\mathrm{T}}$. This approach is found to give excellent performance, and to result in a stable jet mass distribution from 2012 pile-up conditions [10] up to an average of 200 collisions per bunch crossing [11].

\section{Jet calibration}

After building jets, they have to be calibrated to account for several effects. The calibration procedure consists of many steps, as outlined in Figure 2, but can be split into three primary stages: first the pile-up contributions are suppressed at the jet level (for small- $R$ jets), then the jet is calibrated to the Monte Carlo (MC) truth scale, and finally the differences between MC and data are accounted for.

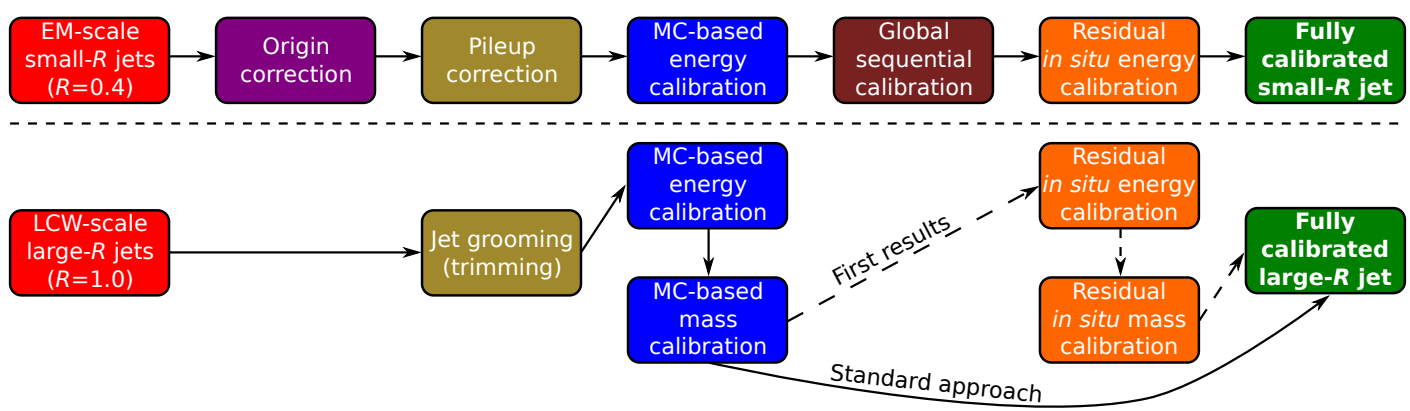

Figure 2. An overview of the calibration chain for both small- $R$ and large- $R$ jets is shown. The dashed line for large- $R$ jets represents preliminary results that are not yet used in physics analyses, while the solid line represents the standard approach that is typically used.

\subsection{Pileup suppression}

Before calibrating jets, typically with multiplicative scale factors, it is important to first remove the contribution from pile-up sources. As explained in Section 3.3, this is done using grooming for large$R$ jets, which is a jet-by-jet means of removing pile-up topo-clusters from an existing jet. Small- $R$ jets follow a different approach, which itself comes in a few steps.

First, the jet axis is corrected to point to the hard-scatter vertex of interest, which is called the origin correction. Then, the event-by-event energy density $\rho$ is calculated within $|\eta|<2.0$ [12], providing a measure of the global pile-up energy present in a given event. This is then used together 
with the jet area $\mathcal{A}_{\text {jet }}$, as evaluated for each individual jet in each event, providing a measure of the local size of the jet. This energy is subtracted from the original jet four-vector to remove the pile-up energy contribution. While this works well in the region where $\rho$ is calculated, it needs some help, especially in the region $|\eta|>2.0$. In this case, average correction factors are derived for a given dataset, parametrized as a value which is differential in the number of reconstructed primary vertices in the event $N_{\mathrm{PV}}$ and the average number of interactions per bunch crossing $\mu$. The final correction is then defined as:

$$
p_{\mathrm{T}}^{\text {corr }}=p_{\mathrm{T}}-\rho \mathcal{A}_{\mathrm{jet}}-\alpha\left(N_{\mathrm{PV}}-1\right)-\beta \mu,
$$

where $\alpha$ and $\beta$ are the correction factors. The impact of each step is shown in Figure 3.

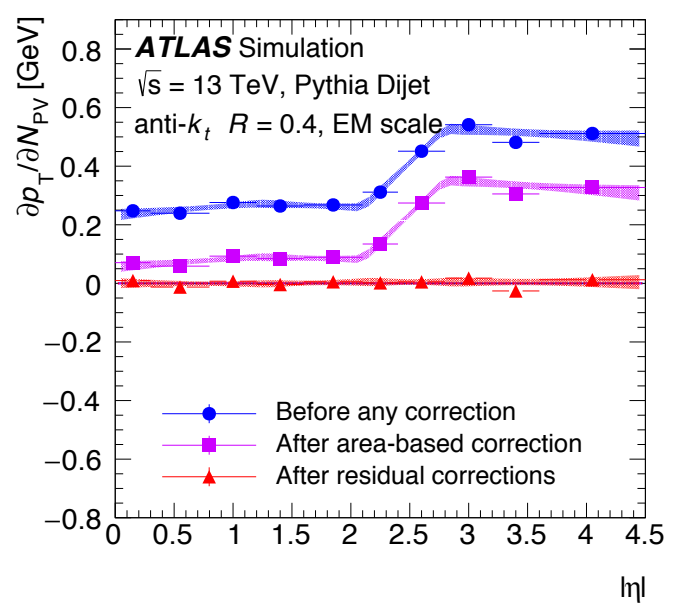

Figure 3. The average amount of pile-up energy added to a small- $R$ jet, per reconstructed primary vertex $N_{\mathrm{PV}}$, is shown. The blue line is for uncorrected jets, the purple line is after the $\rho \mathcal{A}_{\text {jet }}$ area correction, and the red line is after the residual $\alpha$ and $\beta$ corrections [12].

\subsection{MC calibration}

After removing the contribution of pile-up, the next step is to calibrate the jet using MC truth information. This is important because a large fraction of the energy in a hadronic shower is invisible to the detector. As one example, some of the hadronic shower energy will end up in volumes of the detector which are not active sensors, especially where there are cracks. Additionally, hadronic showers inherently rely on interactions with the strong force to start electromagnetic cascades, typically via the creation of $\pi^{0}$ and $\pi^{ \pm}$. However, these strong interactions result in a substantial amount of energy going into splitting nuclei, and thus overcoming the binding energy. This energy is not observed, and must be recovered to accurately measure the Jet Energy Scale (JES).

This can be done in MC simulation by comparing reconstructed jets to jets built from simulated particles, typically referred to as truth jets. By defining the response $X_{\text {reco }} / X_{\text {truth }}$, it is possible to correct for the lost energy, thereby calibrating the average reconstructed jet to the truth scale. Doing this in fine bins of both $\eta$ and energy allows for a correction which properly accounts for both the detector structure and the evolution of hadronic interactions as a function of energy. This same approach is followed for the energy response for both small- $R$ and large- $R$ jets, where the small- $R$ jet energy response (the numerical inverse of the JES calibration factor) is shown in Figure 4.

Large- $R$ jets also ideally have a well-defined mass, as they are used to represent the hadronic decay of a massive particle. It is thus desirable for the large- $R$ jet to have a mass corresponding to the parent massive particle to aid in the identification and interpretation of hadronic events. The same procedure 


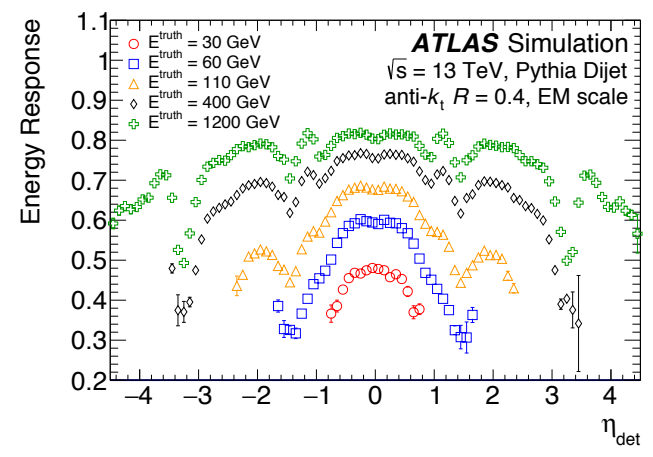

Figure 4. The energy response, $E_{\text {reco }} / E_{\text {true }}$, for small- $R$ jets. The numerical inverse of this response calibrates the average jet back to the truth scale [12].

of matching truth to reconstructed jets is followed, just with the mass response rather than energy and parametrized in $p_{\mathrm{T}}$, mass, and $\eta$ [13]. This is referred to as the Jet Mass Scale (JMS) calibration.

Small- $R$ jets further refine the JES by the application of the Global Sequential Calibration (GSC), which use further jet responses for properties related to shower development, flavour differences, and punch-through (jets which are not fully contained by the calorimeters) [14]. These further refinements are designed to not change the central value of the JES, excluding the punch-through correction, but rather to only reduce the differences between different populations. This thereby results in a better Jet Energy Resolution (JER), which corresponds to a narrower width jet energy distribution.

\subsection{In situ calibration}

Following the application of the JES and JMS calibrations as appropriate, it is important to understand how well the calibration factors are modelled in data. These are purely data-derived calibrations, and thus any differences from data can lead to biases in the jet energy (or $p_{\mathrm{T}}$ ) and mass. Historically, large- $R$ jets in ATLAS have addressed this concern by using $\mathcal{R}_{\text {trk }}$ [13], where

$$
\mathcal{R}_{\text {trk }}=\frac{\left(X_{\text {calo }} / X_{\text {track }}\right)_{\text {data }}}{\left(X_{\text {calo }} / X_{\text {track }}\right)_{\mathrm{MC}}} .
$$

This double-ratio quantifies the differences between the tracker and calorimeter representations of jets, thereby using the well-understood tracker to provide uncertainties for the calorimeter observable $X$ of interest. This technique is useful as it is simple and can provide uncertainties on any calorimeter-based variable, but it only provides an uncertainty on differences between data and MC.

Instead of using the differences as an uncertainty, an in situ calibration can correct for the data vs MC differences. This has been done since the 2011 dataset [15] for small- $R$ jets, and in a restricted manner for large- $R$ jets in the 2012 dataset [13]. However, it has been further expanded for the large- $R$ JES and done for the first time for the JMS with the 2016 dataset [16].

The small- $R$ jet in situ calibration has been refined over many iterations. The first step is the dijet $\eta$-intercalibration, where well-understood central jets are successively used to calibrate more forward jets. This exploits the $p_{\mathrm{T}}$ balance between the jets in dijet events and provides a relative correction between jets as shown in Figure 5, thereby flattening the response across the detector. This is followed by a series of $p_{\mathrm{T}}$ balance methods where well-understood reference objects are used to correct the absolute scale of jets between data and MC. This is done first with $Z+$ jets and $\gamma+$ jets events, where $Z \rightarrow \ell^{+} \ell^{-}$, for $\ell \in\{e, \mu\}$. This provides correction factors from roughly $20 \mathrm{GeV}$ to $1 \mathrm{TeV}$ of $p_{\mathrm{T}}$ [17]. To extend this to even higher energy scales, the multi-jet balance procedure is used, where events with a single high- $p_{\mathrm{T}}$ jet balanced by multiple low- $p_{\mathrm{T}}$ jets within the kinematic range of the $Z+$ jet and 
$\gamma+$ jet calibrations are used. This procedure is iterated, ultimately providing correction factors from roughly $200 \mathrm{GeV}$ to $2 \mathrm{TeV}$ of $p_{\mathrm{T}}$. Finally, the $Z+$ jet, $\gamma+$ jet, and multi-jet balance calibration factors are all combined to define the final small- $R$ jet in situ calibration, shown in Figure 6.

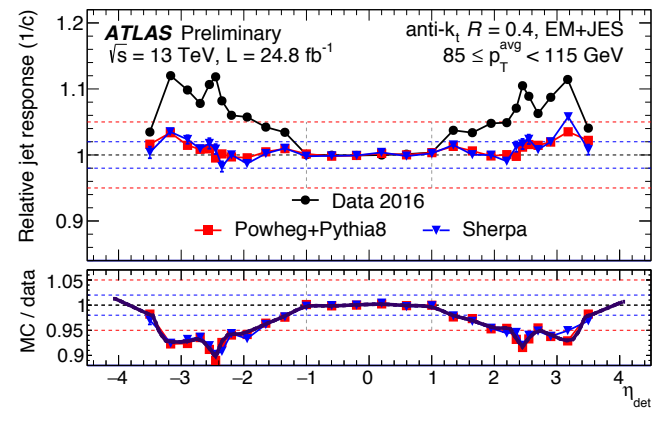

Figure 5. The results of the dijet $\eta$-intercalibration for one $p_{\mathrm{T}}$ bin, showing the dependence of the small- $R$ jet response across $\eta$ and how it differs between data and MC. The thick solid line in the lower box is the final in situ relative calibration curve [17].

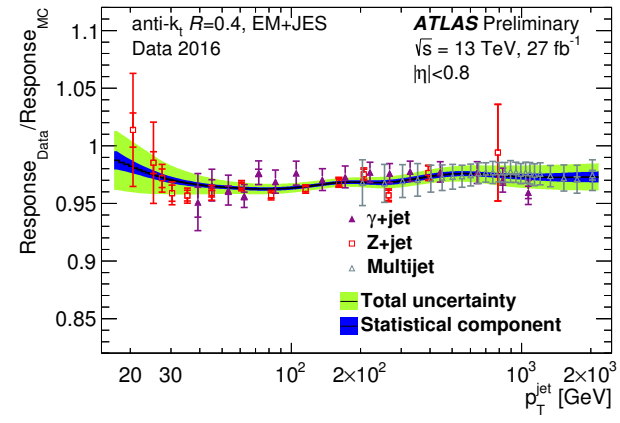

Figure 6. The results of the $Z+$ jet, $\gamma+$ jet, and multi-jet balance in situ calibrations, quantifying the large- $R$ jet difference between data and MC as a function of $p_{\mathrm{T}}$. The black line within the blue band is the final in situ absolute calibration curve [17].

The large- $R$ jet procedure follows a similar approach, except that only the $\gamma+$ jet and multi-jet balance techniques have been performed so far for the JES, as well as their combination [16], as shown in Figure 7. The mass has also been studied in situ using the forward folding method, where high-purity samples of hadronically decaying $W$-bosons and top-quarks are used to constrain the JMS scale in both data and MC. This procedure is significantly limited by the available statistics, and thus is only done in a restricted kinematic range. The $\mathcal{R}_{\text {trk }}$ procedure is used to extend the in situ JMS calibration, where a combination of the two methods is ultimately used as shown in Figure 8.

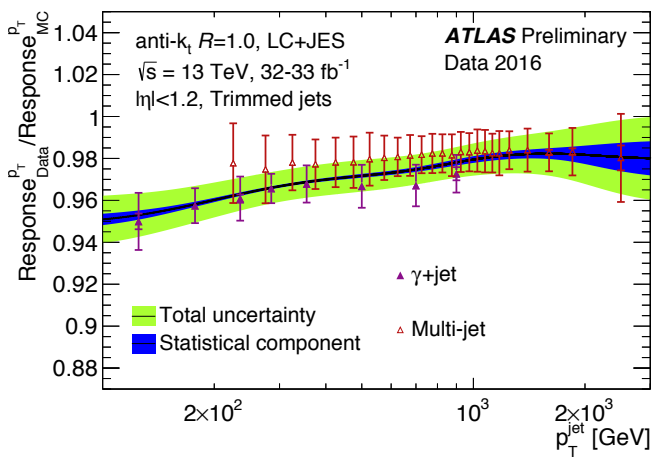

Figure 7. The results of the $\gamma+$ jet and multi-jet balance in situ $p_{\mathrm{T}}$ calibrations, quantifying the large- $R$ jet difference between data and MC as a function of $p_{\mathrm{T}}$. The black line within the blue band is the final in situ absolute $p_{\mathrm{T}}$ calibration curve [16].

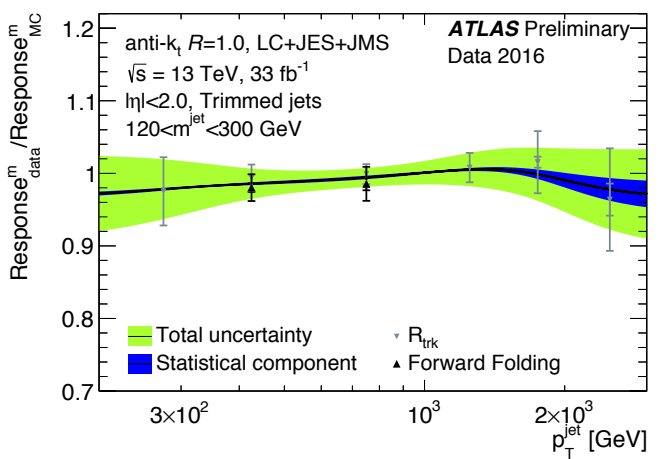

Figure 8. The results of the forward folding and $\mathcal{R}_{\text {trk }}$ in situ mass studies, quantifying the difference between data and $\mathrm{MC}$ as a function of $p_{\mathrm{T}}$ for large- $R$ jets with a top-quark-like mass. The black line within the blue band is the final in situ mass calibration curve [16]. 
These in situ calibration techniques provide very precise results, and have reduced the jet uncertainties substantially. Small- $R$ jets now have a JES uncertainty at or below $1 \%$ from $100 \mathrm{GeV}$ to $1 \mathrm{TeV}$ [17], while large- $R$ jets have a JES uncertainty from $1-2 \%$ from $200 \mathrm{GeV}$ until $2 \mathrm{TeV}$ instead of the $2-3 \%$ uncertainty from $200 \mathrm{GeV}$ to $1 \mathrm{TeV}$ that they have when using only the $\mathcal{R}_{\text {trk }}$ approach [16].

\section{Jet tagging}

Once jets have been calibrated, the next step is to start looking at their substructure, meaning the angular distribution of energy within the jet. This is very useful in identifying the origin of the jet, and particularly to separate boosted massive hadronic particle decays from QCD dijet/multijet production. The most intuitive substructure variable, the jet mass, has already been discussed in the context of calibration. However, the mass of a jet built from topo-clusters can be further refined by including tracking information in the form of the track-assisted mass, $m_{\mathrm{TA}}=m_{\text {track }} \times \frac{p_{\mathrm{T}}^{\text {calo }}}{p_{\mathrm{T}}^{\text {track }}}$. This contains complementary information to the calorimeter mass, $m_{\text {calo. }}$. As such, a linear combination of the two named the combined mass, $m_{\mathrm{comb}}$, is even more useful:

$$
m_{\mathrm{comb}}=A m_{\mathrm{calo}}+B m_{\mathrm{TA}} \quad \text { for } \quad A=\frac{\sigma^{-2}\left(m_{\text {calo }}\right)}{\sigma^{-2}\left(m_{\text {calo }}\right)+\sigma^{-2}\left(m_{\mathrm{TA}}\right)} \quad \text { and } \quad B=\frac{\sigma^{-2}\left(m_{\mathrm{TA}}\right)}{\sigma^{-2}\left(m_{\text {calo }}\right)+\sigma^{-2}\left(m_{\mathrm{TA}}\right)},
$$

where $\sigma\left(m_{x}\right)$ is the Jet Mass Resolution (JMR) for mass definition $x$ [18]. This definition substantially improves the JMR, which is very important for jet tagging purposes, as it impacts the level of separation between different physical processes. This is demonstrated in Figure 9, where the QCD, $W$-jet, and top-jet combined mass distributions are shown. By making the peaks sharper, the amount of background entering a given signal peak would be reduced.

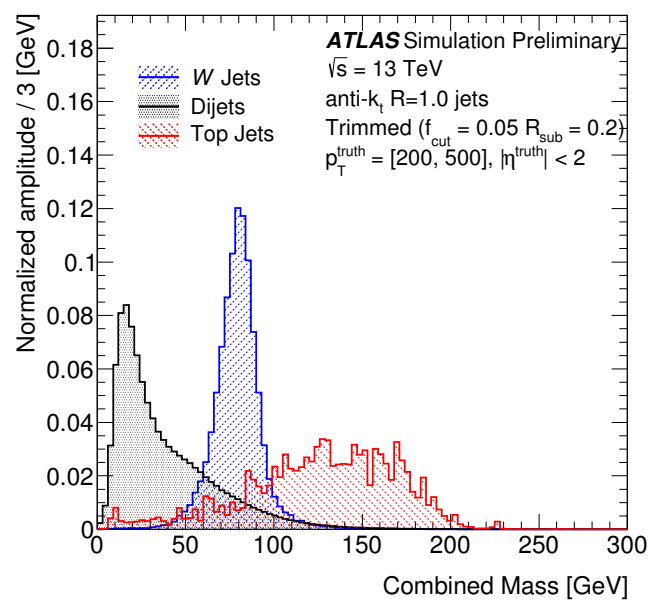

Figure 9. The combined jet mass, $m_{\text {comb }}$, is a linear combination of calorimeter and track-assisted jet masses as defined in the text. It provides good separation between QCD jets, $W$-boson jets, and top-quark jets [19].

While the jet mass is a very powerful substructure variable for discriminating between QCD jets and hadronically decaying massive particles, it is not the only such variable. The best second variable to use depends on the process of interest, and thus different combinations are used for the identification of $W$-bosons in Figure 10 and top-quarks in Figure 11. In addition to two-variable taggers, Boosted Decision Trees (BDTs) and Deep Neural Networks (DNNs) have been used with a set of roughly 10 jet substructure variables each. The $W$-tagging performance is evaluated for a fixed $50 \%$ signal efficiency 
working point, while the top-tagger uses a fixed $80 \%$ signal efficiency working point. In both cases there are gains from using more advanced taggers rather than two-variable selections, although the relative importance varies. In the case of the $W$-tagger, there is a gain of roughly $25 \%$ from using a BDT or DNN, while the top-tagger improves by a factor of 2 with such techniques [19].

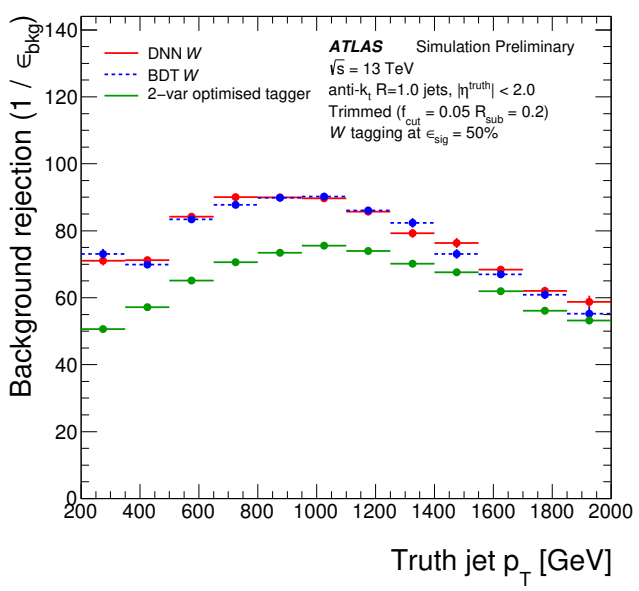

Figure 10. Comparisons of the $W$-tagging performance at fixed $50 \%$ signal efficiency using twovariable optimized taggers or Boosted Decision Trees (BDTs) and Deep Neural Networks (DNNs) built with roughly 10 substructure variables [19].

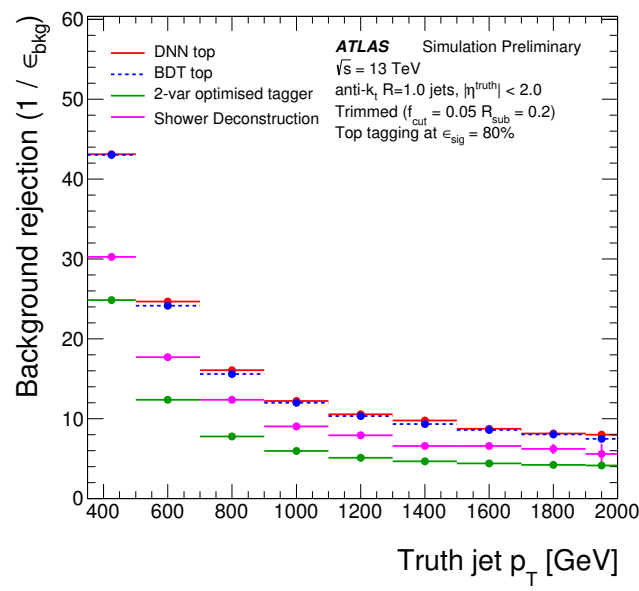

Figure 11. Comparisons of the top-tagging performance at fixed $80 \%$ signal efficiency using twovariable optimized taggers or Boosted Decision Trees (BDTs) and Deep Neural Networks (DNNs) built with roughly 10 substructure variables [19].

$H$-tagging ( $H \rightarrow b b$ identification) relies primarily on $b$-tagging to suppress the large QCD background. However, further cuts on the jet mass and other substructure variables can bring sensitivity improvements [20]. In this case, the signal efficiency is not constant, rather the $H$-tagging efficiency for different selections is shown in Figure 12. In terms of QCD rejection, one $b$-tag provides a background rejection factor of roughly 50, two $b$-tags increases that to 200, a mass cut further improves the rejection to 600, and an additional substructure cut results in a QCD rejection factor of over 1000 [20].

The quark vs gluon tagger is very different from the start, as it uses small- $R$ jets rather than large- $R$ jets. Additionally, the jet mass is not useful, as gluons are massless, and non-top-quarks are effectively massless at the LHC. Instead, the track multiplicity provides the majority of the possible separation power, and the jet width provides most of the remaining discrimination between quarks and gluons [21]. By building a Convolutional Neural Network (CNN) using jet image representations of the jet calorimeter and tracking constitutents, this can be further improved, with some gains for high quark-tagging efficiencies as seen in Figure 13.

\subsection{Future tagging developments}

In order to further improve jet tagging performance, it is important to understand the current limitations. For $W$-tagging, there is a clear convergence of the different techniques at very high $p_{\mathrm{T}}$, as seen in Figure 10. This is because the calorimeter granularity becomes insufficient to resolve the spatial separation between the pair of quarks from a $W$ decay in such boosted events, as the jet collimation starts to fall within a single calorimeter cell. By exploiting the excellent spatial resolution of 


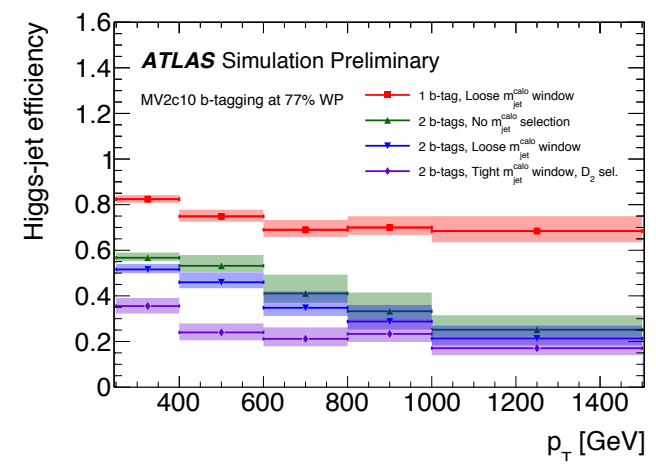

Figure 12. The $H$-tagging signal efficiency for subsequent tagging cuts. Corresponding QCD jet rejection factors for each option are listed in the text [20].

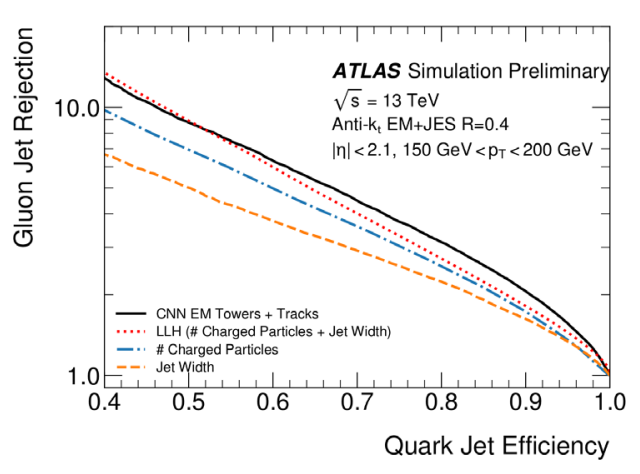

Figure 13. The non-top-quark vs gluon discrimination performance comparing the number of tracks (blue), jet width (yellow), their combination (red), and a Convolutional Neural Network (CNN, black) [21].

the tracker and the excellent energy resolution of the calorimeter in highly boosted jets, it is possible to circumvent this detector constraint. A new type of particle flow designed specifically for jet substructure and jet tagging, referred to as TrackCaloClusters (TCCs), shows excellent performance in Figure 14 when reconstructing the most important $W$ - and $Z$-tagging variable after the jet mass [22].

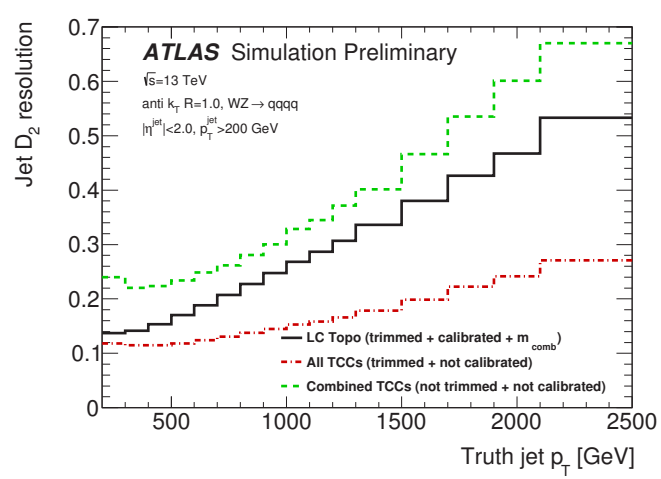

Figure 14. A comparison in $W$ and $Z$ boson jets of the normal calorimeter (black) and TrackCaloCluster (TCC, red) resolutions for the most important $W$ - and $Z$-tagging variable after the jet mass [22].

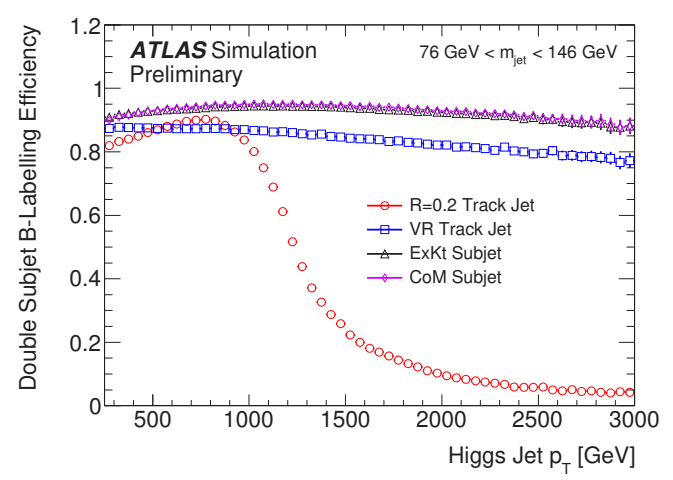

Figure 15. A comparison in $H \rightarrow b b$ jets of the truthlevel labelling efficiency for double-subjets with different subjet reconstruction techniques, showing the need to avoid using fixed $R=0.2$ subjets (red) [23].

For $H$-tagging, a similar problem is seen for very boosted events in Figure 12 . The $H$-tagging efficiency is seen to drop rapidly with $p_{\mathrm{T}}$ as soon as two $b$-tags are required, reducing the usefulness of the current approach for the most energetic events. This is due to the use of $R=0.2$ track-jets for the double $b$-tagging, where at such high energies the two $b$-quarks from the $H$ boson decay are merging or merged [23]. As a result, the $H$-tagging efficiency drops drastically even at the truth-level, 
and new subjet reconstruction techniques are needed for the double $b$-tagging, as shown in Figure 15. These techniques are very promising as a way to recover high- $p_{\mathrm{T}} H \rightarrow b b$ tagging efficiency.

\section{Conclusions}

Jet reconstruction, calibration, and tagging are key pieces of the LHC physics program, and are major areas of research within the ATLAS collaboration. Small- $R$ jets are used to represent non-top-quarks and gluons, and to differentiate between the two. Large- $R$ jets are instead used to identify the decays of massive hadronically decaying particles, such as $W$ and $Z$ bosons, $H \rightarrow b b$, and top quarks.

The small- $R$ jet calibration procedures continue to be refined, providing uncertainties at or below the level of $1 \%$ on the jet energy scale from $100 \mathrm{GeV}$ to $1 \mathrm{TeV}$. Many of these techniques have now also been used for large- $R$ jets, similarly improving the uncertainties, in addition to new methods which providing a first in situ constraint on the jet mass.

The tagging of $W$ bosons and top quarks has been investigated using Boosted Decision Trees and Deep Neural Networks, which are shown to provide improvements to the background rejection by $25 \%$ for $W$ bosons and $100 \%$ for top quarks. Further refinements to tagging procedures by defining a new substructure-oriented particle flow for $W / Z$ boson tagging and a new subjet reconstruction procedure for $H \rightarrow b b$ tagging provide a positive outlook for the future of boosted jet tagging.

\section{References}

[1] L. Evans, P. Bryant, JINST 3, S08001 (2008)

[2] ATLAS Collaboration, JINST 3, S08003 (2008)

[3] ATLAS Collaboration, Eur. Phys. J. C77, 490 (2017), 1603.02934

[4] ATLAS Collaboration, ATLAS-CONF-2017-065 (2017)

[5] M. Cacciari, G.P. Salam, G. Soyez, JHEP 04, 063 (2008), 0802 . 1189

[6] M. Cacciari, G.P. Salam, G. Soyez, Eur. Phys. J. C 72, 1896 (2011)

[7] ATLAS Collaboration, JHEP 09, 076 (2013), 1306. 4945

[8] D. Krohn, J. Thaler, L.T. Wang, JHEP 02, 084 (2010), 0912 . 1342

[9] S.D. Ellis, D.E. Soper, Phys. Rev. D48, 3160 (1993), hep-ph/9305266

[10] ATLAS Collaboration, Eur. Phys. J. C76, 154 (2016), 1510.05821

[11] ATLAS Collaboration, Jet substructure at very high luminosity (2014), https: //twiki.cern.ch/twiki/bin/view/AtlasPublic/JetSubstructureECFA2014/

[12] ATLAS Collaboration, Phys. Rev. D96, 072002 (2017), 1703.09665

[13] ATLAS Collaboration, ATLAS-CONF-2015-037 (2015)

[14] ATLAS Collaboration, ATLAS-CONF-2015-002 (2015)

[15] ATLAS Collaboration, Eur. Phys. J. C75, 17 (2015), 1406.0076

[16] ATLAS Collaboration, ATLAS-CONF-2017-063 (2017)

[17] ATLAS Collaboration, JES Public Plots for Moriond 2017 (2017), https://atlas . web.cern.ch/Atlas/GROUPS/PHYSICS/PLOTS/JETM-2017-003/

[18] ATLAS Collaboration, ATLAS-CONF-2016-035 (2016)

[19] ATLAS Collaboration, ATLAS-CONF-2017-064 (2017)

[20] ATLAS Collaboration, ATLAS-CONF-2016-039 (2016)

[21] ATLAS Collaboration, ATL-PHYS-PUB-2017-017 (2017)

[22] ATLAS Collaboration, ATL-PHYS-PUB-2017-015 (2017)

[23] ATLAS Collaboration, ATL-PHYS-PUB-2017-010 (2017) 